\title{
PIGGYBACK CROWDSENSING: NOVEL APPROACH FOR COLLECTING SENSED DATA
}

\author{
Pranjal Naringrekar ${ }^{1}$, Nisha Chawda ${ }^{2}$, Swapna Naik $^{3}$ \\ ${ }^{1}$ Diploma in Computer Engineering, Shri Bhagubhai Mafatlal Polytechnic, Mumbai, 4/C-6, New Shalimar Apt, \\ JKNagar, Malad(W), Mumbai-95 \\ ${ }^{2}$ Diploma in Computer Engineering, Shri Bhagubhai Mafatlal Polytechnic, Mumbai, 804/C, NeelYog Tower, \\ Dhanjiwadi, Malad(E), Mumbai-97 \\ ${ }^{3}$ Sr. Lecturer, Information Technology Department, Shri Bhagubhai Mafatlal Polytechnic, Mumbai, 304/23-C, \\ Himalaya Vihar, Near Shetty School, Powai, Mumbai-76
}

\begin{abstract}
This paper proposes a task allocation framework called icrowd also known as crowdtasker for mobile crowdsensing using energy efficient piggyback crowdsensing. This task uses sensor readings taken from participants of each area which is further divided into sub-areas by the organizer. Crowdtasker will predict the call and the location of mobile users and set the users according to required goals. The piggyback crowdsensing uses the sensing cycles for collection of data. The users used in sensing tasks are divided into two types that is data plan users and non-data plan users. The data plan users use $3 G$ calls while non-data plan users use Bluetooth gateway for uploading the sensed data.
\end{abstract}

Keywords- Mobile Crowdsensing, Task Allocation, Data Uploading, Sensing Cycles $* * *$

\section{INTRODUCTION}

Piggybacking is a data transmission technique using bidirectional mode in the network layer. It sends data frames from receiver to sender and adds the confirmation that the data sent by the sender was received by the receiver successfully. This means, that instead of sending an acknowledgement in a frame it is piggy-backed on the data frame[1].

Crowdsensing means sharing data collected by sensing devices, which is used for common interest. Hence, piggyback crowdsensing refers to transmit data bidirectionally for collection of shared data gathered by sensing devices [2]. As the sensor equipped smartphones are trending the way of completing the allocated task of environmental or social monitoring has become effective. Instead of deploying sensor separately for such tasks they are inbuilt in the mobile phones itself and hence the collection of the data through this way is referred to as mobile crowdsensing. To carry out this operation the inconvenience incurred to users should be minimized and therefore a framework designed for this purpose is called as piggyback crowdsensing [2].

In designing the task the server needs to setup for storing the sensed data. While designing these tasks the two important key factors to be remembered are data uploading cost and the energy consumption [3]. Piggyback crowdsensing is a technique which controls these two factors efficiently.

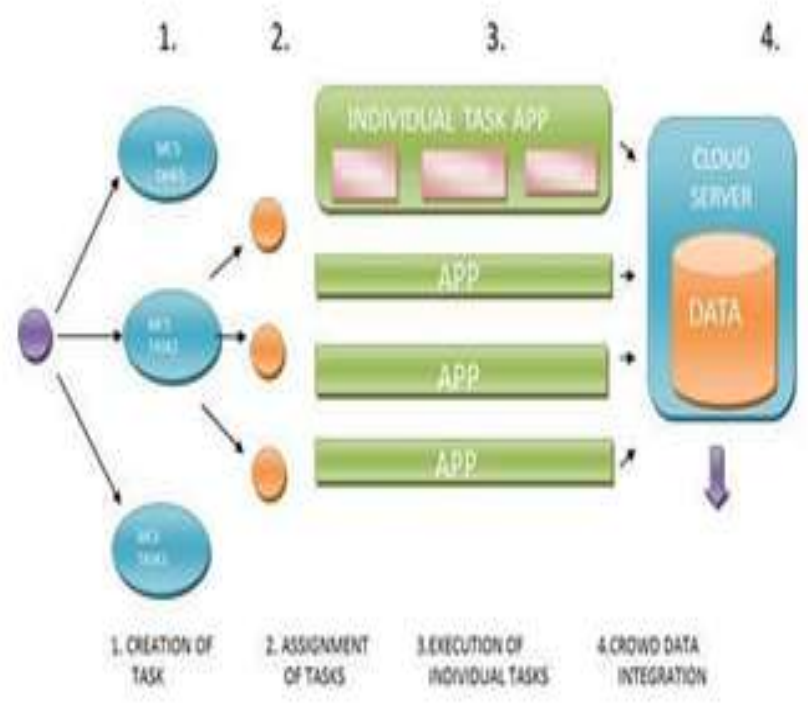

Fig 1: Piggyback Crowdsensing

In piggyback crowdsensing there are two main players that is organizers and participants who carry out the sensing tasks. The organizers are responsible for organizing tasks and the participants are responsible for executing this task. Also, the participants are divided into two categories that is data plan and non-data plan participants [4][6]. 


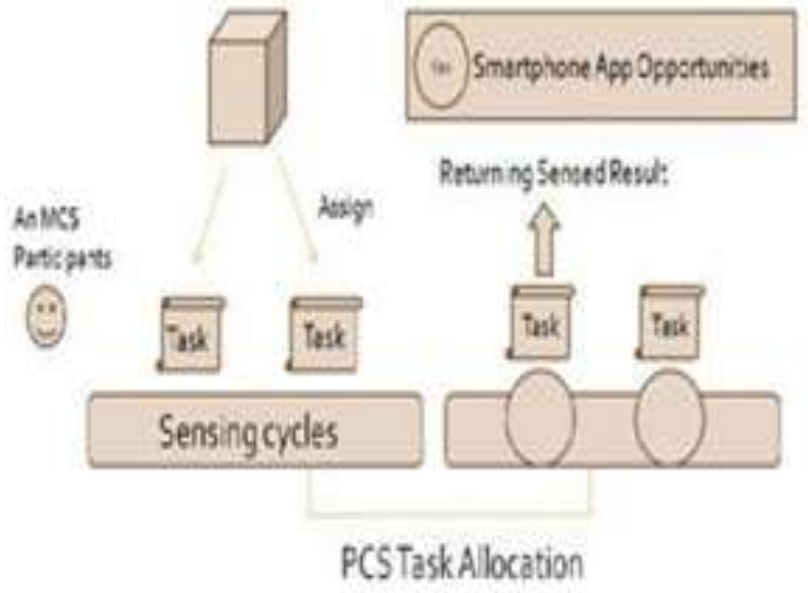

Fig 2: Task Allocation Execution

To carry out these tasks three important datasets are used. The datasets are MIT, NODOBO and D4D. The MIT and NODOBO are used for designing its own dataset for their respective applications. While D4D (data for development) is a dataset having 50,000 call records of the users which include the duration of calls, time of call, time of disconnect [5]. The D4D dataset has its limitations which restricted the other researchers to use the D4D dataset.

\section{RELATED WORK}

Many researches have been carried out for the collection of social or environmental data. We elaborate some of the important technical papers written for data collection.

\subsection{Nodobo}

Nodobo is a set of software developed by google android. Android phones with a special operating system is used to collect data. This software is designed to gather social context data, call logs and text messages. The data is stored on a password secured server. A flat database is used to store data as adding or removing context sensors would be efficient, as normal database would require the creation of new schema [6].

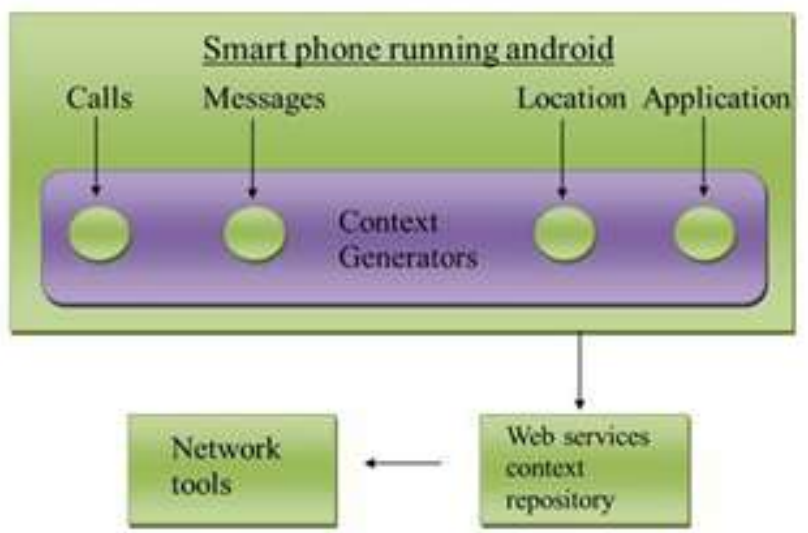

Fig 3: Nodobo Architecture

\subsection{Mobile Crowdsensing}

A framework is designed for data uploading which is energy and cost effective called mobile crowdsensing [6]. As sensor equipped smartphones are becoming popular the mobile crowd sensing has become an effective way to carry out various sensing tasks.

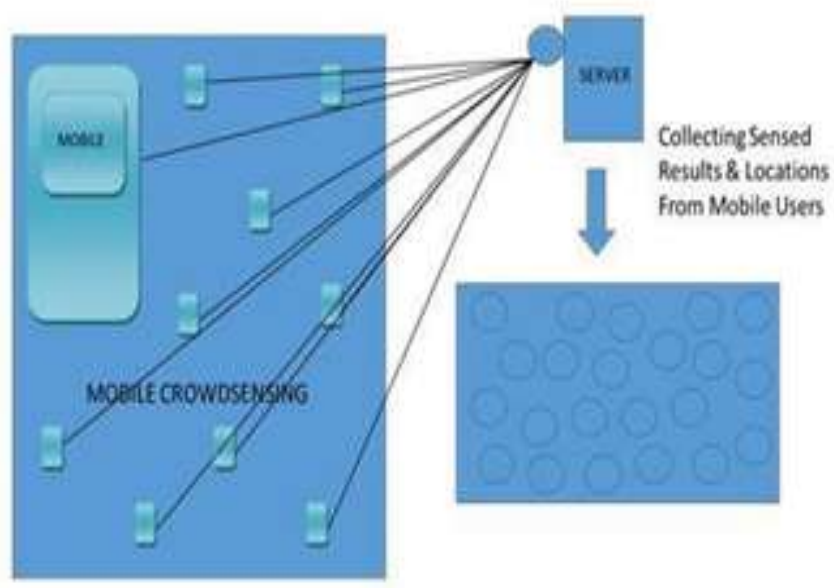

Fig 4: Mobile Sensing

\subsection{Effsense}

Effsense a novel mobile crowd-sensing framework for energy efficient and cost effective data uploading. There are two types of users that is data plan users and non-data plan users which transfers the sensed data to the database based on sensing cycles using appropriate way respectively.

\section{COMPONENTS}

Two important key factors for pcs are:

1. Organizers

2. Participants

Organizers recruit participants for sensing tasks. Organizers define the area for sensing tasks and further divides it into sub-areas. It also determines the sensing cycles. Participants through mobile application using sensor uploads the data to the server. Piggyback crowdsensing uses the Mobile crowd sensing for collecting the sensed reading of the participants. The piggyback crowd sensing makes mobile crowd sensing work efficiently by reducing the energy consumption [4][9]. For example, in a disaster like fire where the temperature rises above the normal temperature, the sensor in the mobile crowd sensing app will sense this rise of temperature and automatically report to the server. The information reported to the server will be used by respective authorities to save the lives of the people.

\section{APPLICATIONS}

There are various examples which are considered to be the domain for collecting the sensed data. A sensor equipped smartphone is used for collection of data. The sensors may be of different types depending on the domain for which the 
data needs to be collected. Some of the widely used sensors are humidity, pressure, temperature, optical, flow, magnetic, position, heartbeat, speed and ultrasonic [8][10].

If the organizer organizes the task of monitoring the temperature of a region then it needs to setup a database, manage sensing cycles and manage the participants and the uploaded data [7]. The organizer will setup a database which will store the sensed temperature data. Organizer will also select the participants having a sensor equipped smartphone with the respective application in the smartphone through which data is uploaded to the server. Organizer will also select the time duration of sensing cycles that is the time after which application on the participant's smartphone will upload the sensed data. The sensing cycles may be of one minute, one hour or one day depending on the need of the organizer. As discussed above there are two types of users that is data plan and non-data plan users. The data plan users will upload the sensed data through the internet or thorough $3 \mathrm{G}$ voice calls while the non-data plan users will upload the data through the Bluetooth gateway. The Bluetooth gateway connects to the nearby data plan users and then send the sensed data to the server [11].

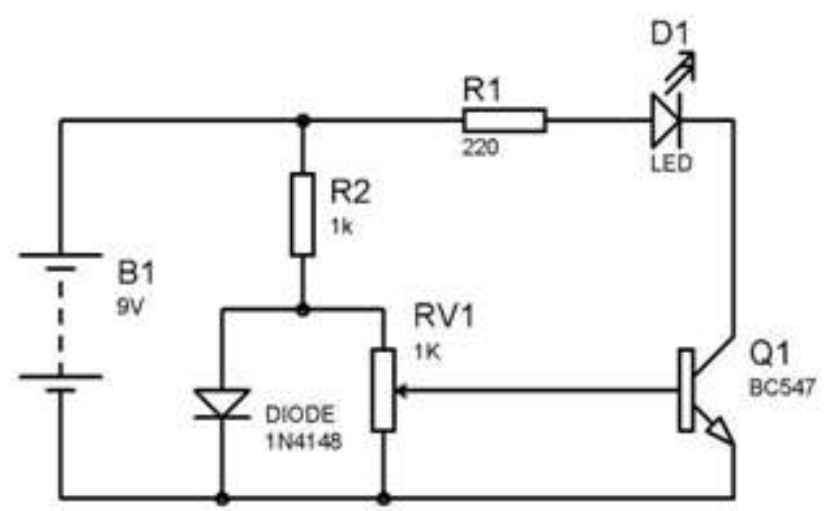

Fig 5: Temperature Sensors

\section{DESIGN ISSUES}

The non-data plan users can upload data with the help of WIFI or send the data to another mobile with the help of Bluetooth which will then forward the data to the server. Hence the uploading cost can be reduced subsequently.

The data plan users can upload data through a $3 \mathrm{G}$ voice call instead of establishing a new $3 \mathrm{G}$ connection. The data can be piggybacked through a voice call. Hence data plan users can reduce energy consumption for uploading data.

Different algorithms are inbuilt along with the application in sensor equipped smartphones. These algorithms decide whether the data should be offloaded or kept for other purposes. Also, these algorithms should be lightweight and executed at faster speed for efficient data uploading.
This is the first work which focuses on reducing data cost as well as energy consumption for uploading data. The piggyback crowdsensing is related with two real world dataset that is MIT and NODOBO.

In the surveys conducted $48 \%$ of the data was uploaded by the by the non-data plan users and $52 \%$ of the data was uploaded by the data plan users which had a sensing cycle of time 24 hours.

\section{EVALUATION}

As two important dataset are considered for evaluating the piggyback crowdsensing that is MIT and NODOBO we will focus on their different patterns for executing the respective tasks.

In an experimental setup for the MIT dataset they choose seven weeks for collecting the sensed data. The organizer chose 71 active users for this task. Out of 71 users, 30 users consumed the largest volume of mobile data while the remaining 41 users uploaded data via WIFI or Bluetooth gateway. Hence 30 users were termed as data plan users while the remaining 41 users were termed as non-data plan users. The organizer decided to keep a time duration of 24 hours to upload this data and this time duration was termed as sensing cycle. Thus, 14 rounds were conducted for uploading this data. This experiment included uploading data via $3 \mathrm{G}$ voice calls and via a Bluetooth gateway.

For the non-data plan users a bluetooth mechanism was used. Any non-data plan user who comes in contact with a data plan user can transfer its data via bluetooth to the data plan user. But we accurately can't predict the time duration of their meet and hence it may happen that they meet for a short duration which isn't sufficient for transferring entire data. Hence the data transferring for a short duration is not considered to be the actual data as it may happen that entire data is not transmitted [9]. In this case also to save energy consumption the bluetooth 4.0 low energy mechanism is used. This is used only in today's smartphones like nexus 5, iPhone $5 \mathrm{~s}$ and iPhone 6.

While carrying out these tasks many other key factors like energy calculation and battery constraints are also considered. If the users phone battery has already reached a low level then he/she is restricted to upload the data. For example if the maximum low level of battery is $20 \%$ then the users having a battery below $20 \%$ are restricted from uploading the data. The users phone battery level was simulated on real time phone usage records including calls, messages, mobile-data usage etc.

An important decision is to be taken of whether data uploading cost should be saved or energy consumption should be reduced. 
For saving the data uploading cost the data plan users are used as they upload data via $3 \mathrm{G}$ calls. For reducing energy consumption the non-data plan users are used.

\section{BLUETOOTH}

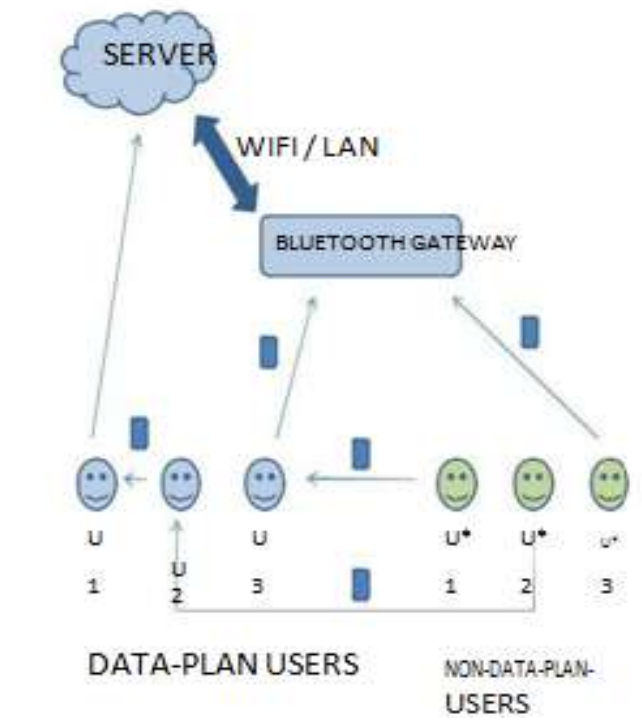

Fig 6: MOBILE CROWDSENSING

Another evaluation carried out was for the algorithms which were used in the applications of sensor equipped smartphones. For this purpose another dataset called D4D (data for development) was used. This dataset has a record of 50,000 users phone call which include time of call, duration of phone call etc. They developed three different methods through which the task could be completed. Later these methods were compared with each other to find the most optimum method for data uploading task [11]. The optimum method was found to upload data through a stationary object as the signal is strongest for stationary objects. There were some limitations with the D4D dataset and hence this caused a limitation of an entire framework [12].

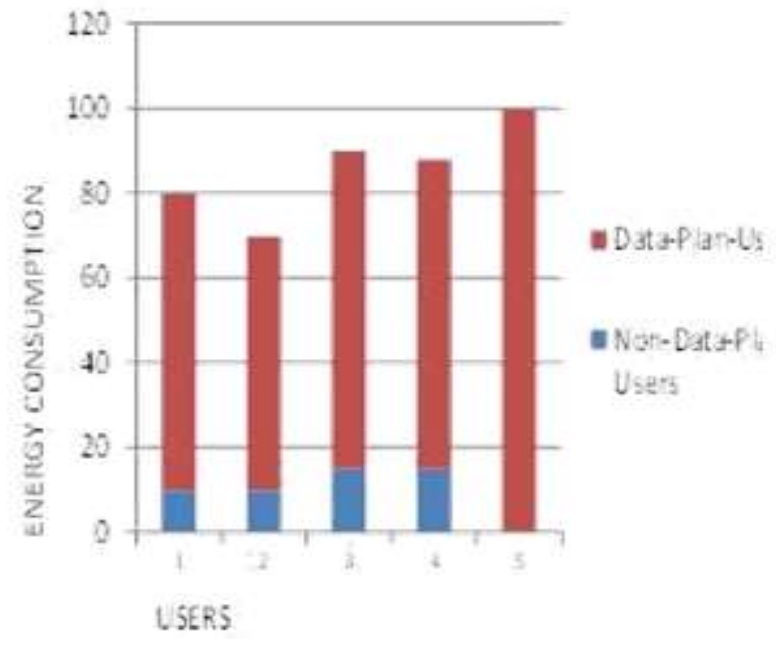

Fig 7: Energy Consumption

\section{FUTURE SCOPE}

The limitations with the papers that were a major part to be taken into consideration which were not acknowledged were security and continuous monitoring of the data bases where the data is uploaded. For improving the security many encrypted methods can be used. Use of cryptography can be done for transferring the sensed data to the data base. Access rights should be defined for each employee. The users should be changed at regular intervals. Also the sensing cycles should also be changed. Different authentication techniques like biometric techniques can be used on users smartphone for avoiding manipulation of the sensed data.

\section{CONCLUSION}

In this paper, we therefore proposed about piggyback crowdsensing - an efficient way for uploading sensed data. This technique used the sensor-equipped smartphones which collected the sensed data with the help of an inbuilt application. There were two types of users that is data plan and non-data plan users. Two important factors that were considered were data uploading cost and energy consumption cost. Three key dataset were used for executing these tasks. They were NODOBO, MIT and D4D.

\section{REFERENCES}

[1] http://ecomputernotes.com/computernetworkingnotes /communication-networks/piggybacking

[2] http://whatis.techtarget.com/definition/pi ggybacking

[3] https://www.computerhope.com/jargon/p/ piggyback.htm

[4] https://www.researchgate.net/publication/ 262257070_Piggyback_CrowdSensing_PCS

_Energy_efficient_crowdsourcing_of_mobil e_sensor_data_by_exploiting_smartphone_a pp_opportunities

[5] https://en.wikipedia.org/wiki/Piggybackin g_(data_transmission)

[6] http://mobilesoa.appspot.com/

[7] https://www.edgefx.in/different-types-of-sensorswith-applications/

[8] https://en.wikipedia.org/wiki/Sensor

[9] https://www.slideshare.net/haoyixiong/ph d-defensetalk-nearoptimal-mobilecrowdsensing-designframework-and-algorithms

[10] http://senda.uab.es/node/15

[11] http://whatis.techtarget.com/definition/d ata-planmobile-data-plan

[12] https://web.njit.edu/ borcea/papers/hand bookcrowdsensing 